\title{
Timing of vessel imaging for suspected large vessel occlusions does not affect groin puncture time in transfer patients with stroke.
}

John W. Liang

Thomas Jefferson University

Laura Stein

Icahn School of Medicine at Mount Sinai

Natalie Wilson

Icahn School of Medicine at Mount Sinai

Johanna T. Fifi

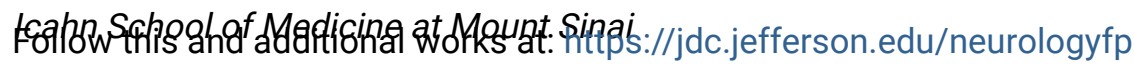

iplay Jfuthinineurology Commons

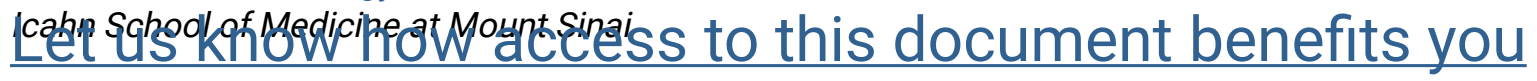

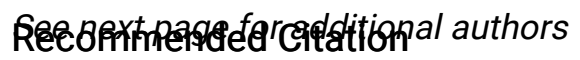

Liang, John W.; Stein, Laura; Wilson, Natalie; Fifi, Johanna T.; Tuhrim, Stanley; and Dhamoon, Mandip S., "Timing of vessel imaging for suspected large vessel occlusions does not affect groin puncture time in transfer patients with stroke." (2018). Department of Neurology Faculty Papers. Paper 147.

https://jdc.jefferson.edu/neurologyfp/147

This Article is brought to you for free and open access by the Jefferson Digital Commons. The Jefferson Digital Commons is a service of Thomas Jefferson University's Center for Teaching and Learning (CTL). The Commons is a showcase for Jefferson books and journals, peer-reviewed scholarly publications, unique historical collections from the University archives, and teaching tools. The Jefferson Digital Commons allows researchers and interested readers anywhere in the world to learn about and keep up to date with Jefferson scholarship. This article has been accepted for inclusion in Department of Neurology Faculty Papers by an authorized administrator of the Jefferson Digital Commons. For more information, please contact: JeffersonDigitalCommons@jefferson.edu. 


\section{Authors}

John W. Liang, Laura Stein, Natalie Wilson, Johanna T. Fifi, Stanley Tuhrim, and Mandip S. Dhamoon 
Timing of vessel imaging for suspected large vessel occlusions does not impact groin puncture time in stroke transfer patients

John W. Liang $\mathrm{MD}^{1,2} \uparrow$; Laura Stein $\mathrm{MD}^{1} \uparrow$; Natalie Wilson $\mathrm{RN}^{1}$; Johanna T. Fifi, MD ${ }^{1,3}$; Stanley Tuhrim MD ${ }^{1}$; Mandip S. Dhamoon MD, DrPH ${ }^{1}$

1. Department of Neurology, Icahn School of Medicine at Mount Sinai, New York, NY, USA

2. Department of Neurology, Divisions of Cerebrovascular Disease, Critical Care and Neurotrauma, Thomas Jefferson University, Philadelphia, PA, USA

3. Department of Neurosurgery, Icahn School of Medicine at Mount Sinai, New York, NY, USA

$\dagger$ Laura Stein and John W. Liang contributed equally to this paper and are co-first authors

\section{Correspondence:}

John W. Liang, MD

Thomas Jefferson University Hospital

Department of Neurology

Divisions of Cerebrovascular Disease, Critical Care and Neurotrauma

909 Walnut Street, 4th floor

Philadelphia, PA 19107

Email: Johnliangmd@gmail.com

Phone: 718-734-7760

\section{Table, 1 Figure}

Abstract: 250

Word count: 1265 
BACKGROUND: Access to endovascular therapy (ET) in acute ischemic stroke may be limited, and rapid transfer of eligible patients to hospitals with endovascular capability is needed. However, the optimal timing of diagnostic CT-angiogram to confirm large vessel occlusion (LVO) is uncertain.

METHODS: Of 57 emergency department transfers to Mount Sinai Hospital (MSH) for possible ET from January 2015 through March 2016, 39 (68\%) underwent ET, among whom 22 (56\%) had CT-angiogram before transfer and 17 (44\%) had CT-angiogram on arrival. We compared mean outside hospital arrival to groin puncture (OTG) time between the two groups using t-tests and Wilcoxon rank sum tests. OTG was defined as the difference between groin puncture and outside hospital arrival time minus ambulance travel time.

RESULTS: Average age was $73 \pm 13$ years and average NIHSS was $19 \pm 5$. There was no difference in average OTG time between the two groups (191 minutes for CT-angiogram at outside hospital vs. 190 minutes for CT-angiogram at $\mathrm{MSH}$ [p=0.99 for t-test and 0.69 for rank sum test]). Among the 18 patients who were transferred but did not receive ET, 10 had no LVO, 5 had large established infarcts on arrival and 3 had post-tissue plasminogen activator hemorrhage. In 9/10 patients without LVO, CT-angiogram was not performed before transfer.

CONCLUSION: CT-angiogram timing in the transfer process does not impact OTG time, but $90 \%$ of patients without LVO had not had CT-angiogram prior to transfer. Hence, it may be beneficial to obtain CT-angiogram at outside hospital, if acquisition and read are rapidly available, to avoid the cost and potential clinical deterioration associated with unnecessary transfers. 



\section{Background}

Multiple randomized controlled trials have demonstrated the superiority of endovascular therapy (ET) in addition to intravenous alteplase (IV-tPA) for eligible patients with confirmed large vessel occlusion (LVO) of the anterior circulation who present within six-hours of symptom onset. As a result, regionalized systems of care are being established for rapid transfer from community hospitals and primary stroke centers to neuro-interventional centers. Studies have demonstrated that rapid transfer of acute stroke patients to neuro-interventional centers for thrombectomy is feasible. ${ }^{1,2}$ Research also suggests that the mean cost of hospitalization for patients transferred for IV-tPA, ET, or both is higher than those directly admitted for the same interventions. ${ }^{3}$ While it is well established that shorter door to groin puncture times result in better outcomes for stroke patients, there is no universally agreed upon transfer protocol.

Despite efforts to utilize clinical stroke scale cutoffs to more quickly triage patients with LVO to neuro-interventional centers, variation in accuracy, predictive value and high false negative rates currently necessitate vascular imaging to confirm LVO. ${ }^{4}$ Holding ambulance crews at the outside hospital (OSH) while waiting to obtain CTangiogram before departure may result in transfer delays. Conversely, not obtaining a CT-angiogram prior to transfer may result in unnecessarily transferring non-LVO patients. To our knowledge, no other studies have examined whether performing CTangiogram prior to or after transfer to a neuro-interventional center results in shorter groin punctures times while controlling for travel time associated with the transfer process. We sought to examine transfer practices and evaluate for differences in groin puncture times in relation to timing of CT-angiogram. We hypothesized that: 1) groin 
puncture times would be similar and 2) transferring patients without CT-angiogram results in the unnecessary transfer of non-LVO cases.

\section{Methods}

Mount Sinai Hospital (MSH) is a 1,171-bed hospital tertiary referral academic medical center in a city of 8.5 million people. As one of 7 hospitals in the Mount Sinai Health System, MSH serves as the receiving hub for endovascular transfers from community emergency departments within our health system and its affiliates. The satellite hospitals within the health system are designated primary stroke centers and physicians from these sending hospitals utilize a 24/7 neurologic emergency hotline to contact the MSH stroke responder on call (stroke fellow, stroke attending or neurocritical care attending). The relevant clinical history, examination and NIHSS are discussed via telephone and the decision to accept the transfer is made by the MSH stroke responder on call. When images are not available a verbal read of the radiologists report is obtained and the images are transferred onto a $\mathrm{CD}$ for transport. Intravenous alteplase is administered, if appropriate, and patients are transferred with alteplase infusing. A centralized transfer center facilitates ambulance transfer, as air transport is not an option. On arrival to MSH, all patients receive a repeat $\mathrm{CTH}$ and, if not already obtained, a CTangiogram.

There were 57 emergency department transfers for ET from January 2015 March 2016. Patients transferred for potential ET but not triaged as an acute stroke at the OSH or transferred from an inpatient service were excluded. We studied the 39 patients who underwent ET. 
Among the 39 ET patients, $22(56 \%)$ had CT-angiogram prior to transfer and 17 (44\%) had CT-angiogram upon arrival at MSH. We calculated ambulance travel time as MSH arrival time minus OSH departure time. To adjust for differences in traffic and travel distances from different sending sites, we calculated OSH arrival to groin puncture (OTG) time as the difference between MSH groin puncture and OSH arrival time minus ambulance travel time (figure). Mean OTG times between the two groups were compared using the t-test and the Wilcoxon rank sum test.

This retrospective analysis was approved by the MSH institutional review board.

\section{Results}

The main cohort consisted of elderly (73 \pm 13 years) patients with severe strokes (NIHSS $19 \pm 5$ ) (Table). There was no significant NIHSS difference between the two groups. Thirty-nine patients $(68 \%)$ transferred with concern for LVO ultimately underwent ET. Of the patients who underwent ET, the average OSH door-to-ambulance pick up time for patients who had CT-angiogram at OSH versus CT-angiogram at MSH was $150 \pm 52 \mathrm{~min}$ (range $79 \mathrm{~min}$ to $286 \mathrm{~min}$ ) and $132 \pm 30 \mathrm{~min}$ (range $93 \mathrm{~min}$ to $206 \mathrm{~min}$ ), respectively. The average travel time for patients who had CT-angiogram at the OSH was $31.4 \pm 14 \mathrm{~min}$ (range $10 \mathrm{~min}$ to $81 \mathrm{~min}$ ) and $34.2 \pm 20 \mathrm{~min}$ (range $11 \mathrm{~min}$ to $90 \mathrm{~min}$ ) for those who had CT-angiogram at MSH. The MSH door-to-groin time was $43 \pm 18$ min for those who received CT-angiogram at $\mathrm{OSH}$ vs $58 \pm 13$ min for those who received CT-angiogram on arrival at MSH. The average OTG time was 191 minutes for those who received CTangiogram at OSH vs. 190 minutes for those who received CT-angiogram on arrival at MSH ( $\mathrm{p}=0.99$ for $\mathrm{t}$-test and 0.69 for rank sum test). Notably, 18 patients (32\%) who were 
transferred did not undergo ET, out of whom 10 had no LVO, 5 had large established infarcts on arrival, and 3 had post-tPA hemorrhage. The 5 patients who had large established infarcts on arrival all had ASPECT $>7$ on presentation at OSH and subsequently worsened on repeat non contrast $\mathrm{CT}$ head at MSH. Among the 16 patients transferred from OSH prior to CT-angiogram, 9 (56\%) were found not to have LVO on CT-angiogram at MSH.

\section{Discussion}

The stroke community has called for regionalized systems of care to expedite equitable administration of IV-tPA and ET for eligible patients. Effective systems of care require collaboration across many parties, including EMS providers, hospital systems, public health agencies, professional societies and guideline makers, as well as data collection and analysis of quality of care. ${ }^{5}$ This analysis shows that, in an organized system, performing CT-angiogram before or after transfer does not appear to significantly impact OTG time but has greater significance for stroke systems of care.

As we move forward into the future of endovascular thrombectomy for acute ischemic stroke, it is important to identify which patients should or should not be transferred for several reasons. First, transferring non-LVO patients requires an ambulance (or helicopters in some institutions) and medical personnel, putting extra demands on our already strained healthcare system. Secondly, moving non-LVO patients to a distant hospital may become a burden on family and friends who wish to visit as well as potentially increasing discharge disposition difficulties. Often, patients receive tPA before transfer and it is unknown if the transfer crew can provide the necessary post-tPA 
care, such as neurological checks and blood pressure control, during the ambulance ride. Given that 10 out of $57(17.5 \%)$ of acute stroke transfers had no LVO and $90 \%$ of the non-LVO cases had not had a CT-angiogram at an OSH, it may be beneficial to obtain CT-angiogram at OSH to avoid unnecessary transfers. Without initial vascular imaging prior to transfer it is not possible to definitively rule out vessel recanalization, either spontaneous or as a result of IV-tPA, in the non-LVO cases. It should be noted that there was no recanalization noted on arrival to MSH in the group who had confirmed LVO on outside vascular imaging. Because recanalization rates in patients who receive IV-tPA are as low as $4-32 \%$, we feel that obtaining the CT-angiogram prior to transfer is still justified. ${ }^{6}$ For all these reasons, it seems reasonable to perform a CT-angiogram to rule-in a potentially treatable LVO before moving patients to a distant hospital.

Other research has suggested that decline in Alberta Stroke Program early CT Scores (ASPECTS) during transfer is associated with worse clinical outcome. ${ }^{7,8}$ Our study further supports a transfer protocol that includes repeating a non-contrast CT scan prior to intervention given the high incidence of established infarcts and post-TPA hemorrhage ( 3 out of 30 tPA patients) found on arrival. Although this additional CT scan exposes the patient to additional radiation, it can be performed rapidly on arrival, does not require additional contrast, and allows for better detection of complications from IVtPA and identification of patients who may no longer benefit from ET after transfer.

This retrospective study is limited by the small number of patients transferred as well as the lack of data on outcomes and costs associated with patients for whom intervention was not performed. We do not have data on CT-angiogram acquisition and interpretation times at both hospitals because that is not routinely and reliably 
documented. Another confounding factor is the imaging protocol variations at different OSH such as creatinine requirement prior to CT-angiogram or ability to obtain CTangiogram immediately after non-contrast CT head. Accounting for these real-life practice variations we looked at different time segments of the entire process and found that those who had CT-angiogram at OSH, compared to those who did not, had longer OSH door-to-ambulance (150min vs 132min), similar ambulance travel (31min vs 34min), and shorter MSH door-to-groin times (43min vs 58min). We postulate that the time differences are due to the time required to obtain and interpret the additional imaging (at the $\mathrm{OSH}$ or at $\mathrm{MSH}$ ).

It seems that patients benefit from repeat $\mathrm{CTH}$ prior to intervention. Our practice to always repeat a noncontrast $\mathrm{CTH}$ on arrival and our streamlined process of getting a subsequent CT-angiogram, if needed, means that the speed of obtaining CT-angiogram at MSH is more predictable and reliable than at the OSH. Although performing CTangiogram prior to transfer does not appear to affect OTG times, it is important to emphasize that the value in obtaining a CT-angiogram at the $\mathrm{OSH}$ is the diagnostic accuracy in not wasting resources associated with transferring non-LVO patients.

Our hospital network has benefited from ongoing collaboration and feedback via semi-annual system-wide symposiums among all stakeholders (emergency department, nursing, administration, transfer personnel, etc) of the acute stroke process in an effort to optimize our workflow. Primary stroke centers accepting potential LVO patients should make efficient CT-angiogram acquisition a priority in acute stroke protocols. However, given the proven superiority of ET, it would be appropriate for centers without access to rapid vascular imaging to transfer suspected LVO patients without CT-angiogram. 
A more reliable prehospital method of triaging patients with suspected LVO may be beneficial to save the time lost by taking patients initially to a primary stroke center before a neuro-interventional center. Further investigation may elucidate patterns in the logistics of the transfer process resulting in established infarcts and post-tPA hemorrhage on arrival such that selection criteria and transfer protocols can be improved.

Additionally, it may be worthwhile to continue to examine cost, length of stay, and complications for patients transferred but ineligible for ET on arrival.

In conclusion, this study provides proof of concept that in an urban, densely populated hospital network, obtaining CT-angiograms at outside hospitals prior to transferring for potential endovascular intervention is feasible. It can help mitigate the transfer of non-LVO patients without causing significant delays in the overall groin puncture time. We advocate focusing resources on streamlining CT-angiogram protocols at primary stroke centers who transfer patients out for endovascular intervention. 
Table 1: Study population characteristics and timing

\begin{tabular}{|l|c|c|c|}
\hline & Total & CTA at OSH & CTA at MSH \\
\hline Number of participants (\%) & 57 & $24(42)$ & $33(58)$ \\
\hline Age (year), mean (SD) & $73.6(13.5)$ & $74.5(14.4)$ & $72.4(12.5)$ \\
\hline NIHSS, mean (SD) & $19.3(5)$ & $18.8(4.8)$ & $20(5.3)$ \\
\hline tPA prior to transfer, number (\%) & 30 & $18(60)$ & $12(40)$ \\
\hline Endovascular Therapy, Number (\%) & 39 & $22(56)$ & $17(44)$ \\
\hline OSH door-to-ambulance, minutes (SD) & $142(44)$ & $150(52)$ & $132(30)$ \\
\hline Ambulance travel, minutes (SD) & $32(17)$ & $31.4(14)$ & $34.2(20)$ \\
\hline MSH door-to-groin, minutes (SD) & $50(18)$ & $43(18)$ & $58(13)$ \\
\hline OTG, minutes (SD) & $190(43)$ & $191(54)$ & $190(25)$ \\
\hline No Endovascular Therapy, Number (\%) & 18 & $2(11 \%)$ & $16(89 \%)$ \\
\hline No LVO, Number (\%) & 10 & $1(10)$ & $9(90)$ \\
\hline Established infarct, Number (\%) & 5 & 0 & $5(100)$ \\
\hline Post tPA hemorrhage, Number (\%) & 3 & $1(33.3)$ & $2(66.7)$ \\
\hline
\end{tabular}

Abbreviations: CTA, CT Angiogram; CTH, CT Head; LVO, Large Vessel Occlusion; MSH, Main Stroke Hospital; NIHSS, National Institute of Health Stroke Scale; OSH, Outside Hospital; OTG, outside hospital door to groin puncture; tPA, Tissue Plasminogen Activator 
Figure: Transfer process work flow

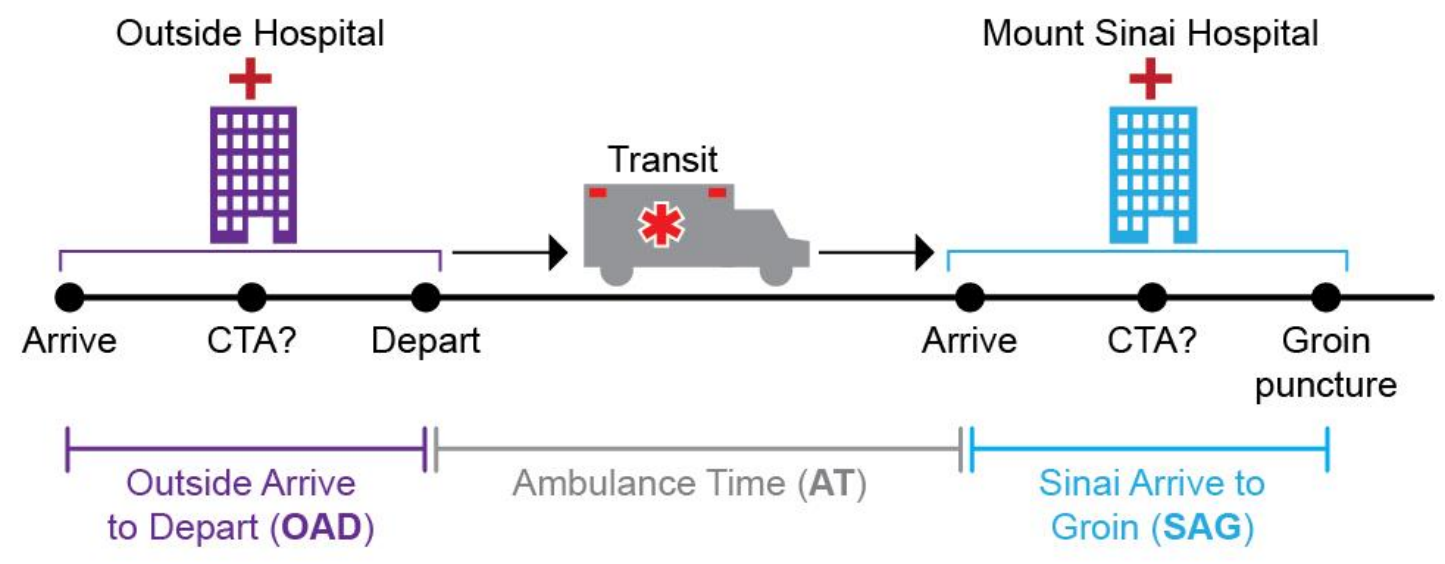

Total time $-A T=$ Outside hospital to groin (OAD+SAG) 


\section{Funding Statement:}

No author has any relevant disclosures.

\section{Competing Interests Statement:}

There are no competing interest

\section{Contributorship Statement:}

LS monitored data collection, and drafted and revised the paper.

JWL initiated the project, designed data collection tools, monitored data collection, drafted and revised the paper.

NW designed data collection tools and monitored data collection, revised the paper JF monitored data collection and revised the paper

ST monitored data collection and revised the paper

MD wrote the statistical analysis plan, cleaned and analysed the data, monitored data collection, and revised the paper. He is guarantor.

Data sharing statement: Data not available on Dryad. 


\section{REFERENCES}

1.Weber R, Reimann G, Weimar C, Winkler A, Berger K, Nordmeyer H, Hadisurya J, Brassel F, Kitzrow M, Krogias C, Weber W, Busch E, Eyding J. Outcome and periprocedural time management in referred versus directly admitted stroke patients treated with thrombectomy. Therapeutic Advances in Neurological Disorders. 2016, Vol. 9(2) 79-84.

2.Pfaff J, Pham M, Herweh C, Wolf M, Ringleb P, Schönenberger S, Bendszus M, Möhlenbruch M. Clinical Outcome After Mechanical Thrombectomy in Non-elderly Patients with Acute Ischemic Stroke in the Anterior Circulation: Primary Admission Versus Patients Referred from Remote Hospitals. Clinical Neuroradiology, 2015, 1-8.

3. Soing A, Lin N, Krishna C, Natarajan S, Mokin M, Hopkins L, Snyder K, Levy E, Siddiqui A. Impact of transfer status on hospitalization cost and discharge disposition for acute ischemic stroke across the US. Journal of Neurosurgery, 2016 May; 124(5):122837.

4. Turc, Guillaume, Benjamin Maïer, Olivier Naggara, Pierre Seners, Clothilde Isabel, Marie Tisserand, Igor Raynouard, et al. "Clinical Scales Do Not Reliably Identify Acute Ischemic Stroke Patients With Large-Artery Occlusion.” Stroke; a Journal of Cerebral Circulation, April 28, 2016. doi:10.1161/STROKEAHA.116.013144.

5. Smith E, Schwamm L. Endovascular clot retrieval therapy: implications for the organization of stroke systems of care in North America. Stroke. 2015 Jun;46(6):1462-7.

6. Bhatia, Rohit, Michael D. Hill, Nandavar Shobha, Bijoy Menon, Simerpreet Bal, Puneet Kochar, Tim Watson, Mayank Goyal, and Andrew M. Demchuk. "Low Rates of Acute Recanalization With Intravenous Recombinant Tissue Plasminogen Activator in Ischemic Stroke Real-World Experience and a Call for Action.” Stroke 41, no. 10 (October 1, 2010): 2254-58. doi:10.1161/STROKEAHA.110.592535.

7. Sun C, Connely K, Nogueira R, Glenn B, Zimmermann S, Anda K, Camp D, et al. ASPECTS Decay during Inter-Facility Transfer Predicts Patient Outcomes in Endovascular Reperfusion for Ischemic Stroke: A Unique Assessment of Dynamic Physiologic Change over Time. Journal of NeuroInterventional Surgery 7, no. 1 (January 1, 2015): 22-26.

8. Mokin M, Gupta R, Guerrero W, Rose D, Burgin W, Sivakanthan S. ASPECTS Decay during Inter-Facility Transfer in Patients with Large Vessel Occlusion Strokes. Journal of NeuroInterventional Surgery, April 22, 2016, neurintsurg-2016-012331. 